\title{
Enantiospecific total synthesis of 6-epi-(-)-hamigeran B. Intramolecular Heck reaction in a sterically constrained environment
}

\author{
Goverdhan Mehta* and Harish M. Shinde \\ Department of Organic Chemistry, Indian Institute of Science, Bangalore 560 012, India
}

\begin{abstract}
An enantiospecific approach, emanating from the abundantly available chiron $R-(+)$-limonene, to the biologically potent, novel marine natural products, the hamigerans has been developed in which an intramolecular Heck coupling between aryl triflates and an alkene serves as the pivotal step. Following this strategy, a synthesis of (-)-6-epi-hamigeran B has been accomplished.
\end{abstract}

Natural products from marine flora and fauna continue to be a rich source of structural diversity and promising biological activity. ${ }^{1}$ In 2000 , Cambie et al. reported the isolation and structure determination of several novel natural products from the poecilosclerid sponge Hamigera tarangaensis BERGQUIST and FROMONT (family Anchinoidae, syn. Phorbasidae) collected from the Hen and Chicken Island off the coast of New Zealand. ${ }^{2}$ Among these natural products, named hamigerans, four compounds, hamigeran A 1, debromohamigeran A 2, hamigeran B 3 and 4-bromohamigeran B 4 are endowed with a unique tricarbocyclic skeleton

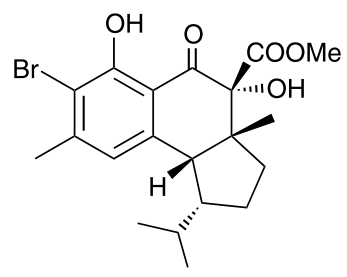

1 Hamigeran A

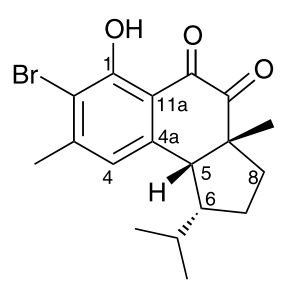

3 Hamigeran B

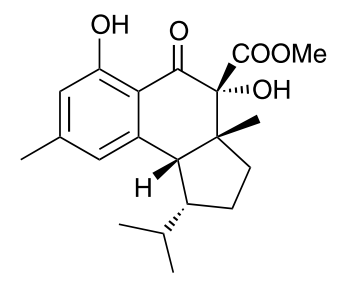

2 Debromohamigeran A

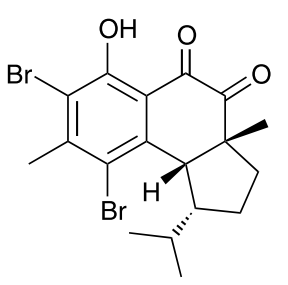

4 4- bromohamigeran B

\footnotetext{
* Corresponding author. E-mail: gm@orgchem.iisc.ernet.in
}

in which a substituted aromatic nucleus is fused to a hydrindane framework bearing three or more stereogenic centres.

Hamigerans are biogenetically quite interesting and it remains to be established whether they are norditerpenoids or meroterpenoids that arise through mixed biogenesis involving a monoterpenoid moiety. The biological activity profile of hamigerans is impressive and some of them exhibit moderate in vitro cytotoxicity against P-388 leukemia cells. However, hamigeran B 3 has been found to be quite exceptional and exhibits $100 \%$ in vitro inhibition against both the herpes and polio viruses, with only slight cytotoxicity. The absolute configuration of hamigeran A $\mathbf{1}$ has been determined by single-crystal X-ray analysis and those of other members of this family inferred from this assignment. ${ }^{3}$ On account of the structural novelty and biological activity, hamigerans constitute challenging synthetic targets and the first total synthesis of these natural products has been accomplished recently by the research group of Nicolaou. ${ }^{4}$ We have been pursuing an enantiospecific route to hamigerans, particularly the biologically most potent hamigeran B 3 from the abundantly available chiron $R$-(+)-limonene 5 and wish to report here the synthesis of 6-epi-hamigeran B 6 following a strategy in which an intramolecular Heck coupling in a sterically compressed environment is the key step.

We have earlier described the restructuring of (+)limonene 5 to the cyclopentane derivative (-)-7, which has proved to be a versatile building block for terpene 

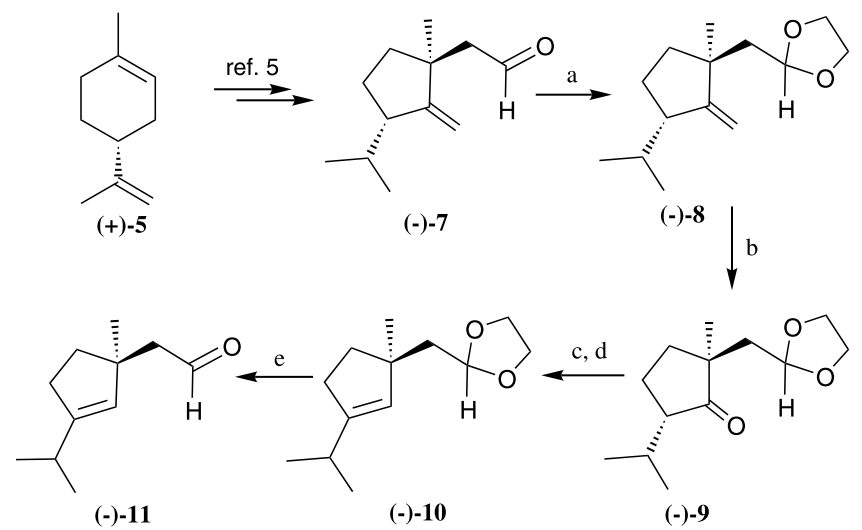

Scheme 1. Reagents and conditions: (a) BTSE, TMSOTf (0.01 equiv.), $\mathrm{DCM},-78^{\circ} \mathrm{C}, 95 \%$; (b) $\mathrm{O}_{3}, \mathrm{MeOH},-78^{\circ} \mathrm{C}$, DMS, $-78^{\circ} \mathrm{C}, 60 \%$; (c) $\mathrm{NaBH}_{4}, \mathrm{MeOH}, 0^{\circ} \mathrm{C}, 95 \%$; (d) $\mathrm{POCl}_{3}$, pyridine, $0^{\circ} \mathrm{C}-\mathrm{rt}, 2 \mathrm{~h}, 50 \%$; (e) $\mathrm{HCl}$, THF, rt, 6 h, 70\%.

synthesis. $^{5}$ The cyclopentane aldehyde $(-)-7^{5}$ with appropriately positioned quaternary methyl and isopropyl groups, with secured stereochemical disposition, appeared to have the structural features that matched very well with the five-membered ring of hamigeran B 3 and thus appeared to be a good precursor for our synthetic pursuit. Protection of the aldehyde group in 7 proved to be unexpectedly tedious but could be achieved using 1,2-bis(trimethylsilyloxy)ethane (BTSE) in the presence of catalytic TMSOTf to furnish the ketal $\mathbf{8}^{6,7}$ Ozonolysis of $\mathbf{8}$ and reductive work-up led to the cyclopentanone 9. Reduction of the carbonyl group and further dehydration of the resulting alcohol transformed 9 to the required endocyclic olefin 10 (Scheme 1). Removal of the protecting group in $\mathbf{1 0}$ delivered the unsaturated aldehyde $\mathbf{1 1}{ }^{7}$
It was planned to generate the tricyclic framework of hamigeran B through an intramolecular Heck coupling ${ }^{8}$ and for that, an appropriate aromatic appendage needed to be installed using the aldehyde group in $\mathbf{1 1}$ as the handle. Directed metalation ${ }^{9}$ of 3(methoxymethoxy)anisole $\mathbf{1 2}$ and addition to $\mathbf{1 1}$ was regioselective and, following pyridinium dichromate (PDC) oxidation, aryl ketone 15 was conveniently obtained (Scheme 2). Similarly, addition of metalated 5-methyl-3-(methoxymethoxy)anisole 13 to 11 and PDC oxidation furnished 16. In another example, metalation of 1-bromo-2-(methoxymethoxy)naphthalene 14, addition to 11, and oxidation furnished $\mathbf{1 7}$ regioselectively (Scheme 2). The directing MOM group in 15-17 was now transformed in two steps to the corresponding triflate functionality ${ }^{10}$ as in $\mathbf{1 8 - 2 0}$, respectively, for implementing the projected intramolecular Heck reaction (Scheme 2). ${ }^{7}$

Substrates 18-20 were now ready for effecting the intramolecular aryl triflate-alkene cyclization ${ }^{8,10}$ and we were cognisant of the sterically compressed nature of the alkene moiety in these compounds. After several trials, it was found that $20 \mathrm{~mol} \%$ of palladium acetate in the presence of $20 \%$ 1,3-bis(diphenylphosphino)propane (DPPP) in DMF effected the contemplated cyclization in 18-20 to furnish 21-23, respectively (Scheme 3). ${ }^{10 \mathrm{~b}}$ While in the case of $\mathbf{1 8}$ and 19. cyclization led to the endocyclic olefinic products $\mathbf{2 1}$ and 22, the naphthalene derivative $\mathbf{2 0}$ furnished the isopropylidene derivative $\mathbf{2 3}$ as the major product with a minor amount of the endocyclic isomer (Scheme 3 ). ${ }^{7}$ In all the cases, products 24-26 of reductive removal of the triflate group from 18-20 were also encountered (Scheme 3). ${ }^{7}$ The cis-stereochemistry at the 6,5-ring junction in 21-23 follows from the X-ray evidence (vide<smiles>COc1cccc(OC)c1</smiles>

12<smiles>COc1cc(C)cc(OC)c1</smiles>

13<smiles>COc1ccc2ccccc2c1Br</smiles>

14

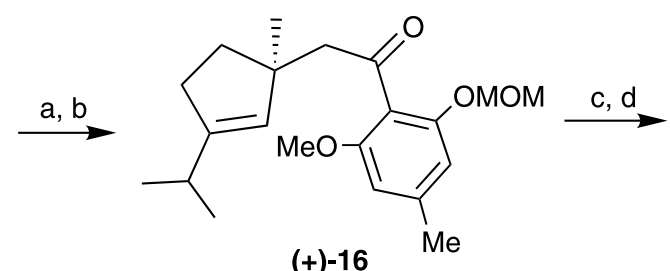

$(+)-16$

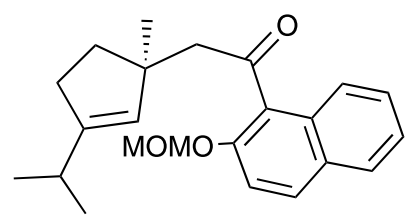

$(+)-17$<smiles>COc1cccc(O)c1C(=O)C[C@@]1(C)CCC(C(C)C)=C1O</smiles>

(+)-18<smiles>COc1cc(C)cc(O)c1C(=O)C[C@]1(C)C=C(C(C)C)CC1</smiles>

(+)-19

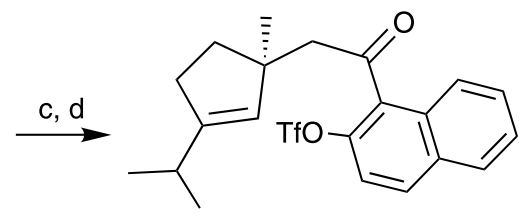

(+)-20

Scheme 2. Reagents and conditions: (a) ${ }^{t} \mathrm{BuLi}$, hexane, $0^{\circ} \mathrm{C}, 1 \mathrm{~h}$ then $-78^{\circ} \mathrm{C}$ to $0^{\circ} \mathrm{C}$; (b) $\mathrm{PDC}, 4 \AA$ mol. sieves, $\mathrm{DCM}, 10 \mathrm{~h}(40 \%$ for two steps); (c) $20 \% \mathrm{HCl}$, THF, $5 \mathrm{~h}, 80 \%$; (d) $\mathrm{Tf}_{2} \mathrm{O}$, pyridine, rt, $10 \mathrm{~h}, 80 \%$; (e) ${ }^{n} \mathrm{BuLi}, \mathrm{THF},-78^{\circ} \mathrm{C}, 1.5 \mathrm{~h}$. 
<smiles>COc1cccc2c1C(=O)C[C@]1(C)CC=C(C(C)C)[C@H]21</smiles>

$(+)-21$

$(2: 1)$<smiles>COc1ccccc1C(=O)C[C@H]1C=C(C(C)C)CC1</smiles>

$(+)-24$<smiles>COc1cc([N+](=O)[O-])cc2c1C(=O)C[C@]1(C)CC=C(C(C)C)C21</smiles>

$(2: 1)$<smiles>COc1cc(C)ccc1C(=O)C[C@]1(C)C=C(C(C)C)CC1</smiles>

$+$<smiles>CC(C)C1=C[C@H](CC(=O)c2cccc3ccccc23)CC1</smiles>

Scheme 3. Reagents and conditions: (a) $\mathrm{Pd}(\mathrm{OAc})_{2}(20 \mathrm{~mol} \%)$, DPPP $\left(20 \mathrm{~mol}^{\%}\right), \mathrm{NEt}_{3}, \mathrm{DMF}, 90^{\circ} \mathrm{C}, 10 \mathrm{~h}, 55-60 \%$.

infra) ${ }^{11}$ and mechanistic considerations. Aryl triflatealkene cyclizations ${ }^{8,10}$ usually proceed through a 'cationic pathway' and after the oxidative addition of the Pd-complex into the carbon triflate bond, faceselective addition to the alkene from the face opposite to the angular methyl group accounts for the observed stereochemistry.

Among the Heck coupling products 21-23, tricycle $\mathbf{2 2}$ had the requisite substitution pattern in the aryl ring for further elaboration to hamigeran B 3. Catalytic hydrogenation of $\mathbf{2 2}$ was stereoselective and a single diastereomer 27 was obtained and its stereostructure was elucidated through X-ray crystal structure ${ }^{11}$ determination (Scheme 4).
Somewhat unexpectedly, hydrogenation of $\mathbf{2 2}$ occurred exclusively from the $\beta$-face to furnish 27 with the C6 isopropyl group being epimeric with respect to hamigeran B 3. Recourse to different catalysts and a change in the solvent regime did not give any evidence of formation of the desired C6 $\beta$-isopropyl isomer. Consequently, we proceeded to complete the synthesis of the C6-epi-hamigeran B 6 (Scheme 4). ${ }^{7}$ Selenium dioxide oxidation of $\mathbf{2 7}$ was clean and delivered $\mathbf{2 8}$ having the requisite $\alpha$-diketone functionality. The methyl ether moiety in $\mathbf{2 8}$ was next deprotected to give 29 with a free hydroxyl group. Finally, bromination of 29 with NBS furnished 6-epi-hamigeran B 6, $[\alpha]_{\mathrm{D}}=$ $-220\left(c 0.2, \mathrm{CHCl}_{3}\right.$ ), having spectroscopic characteristics in full agreement with its formulation. ${ }^{7}$

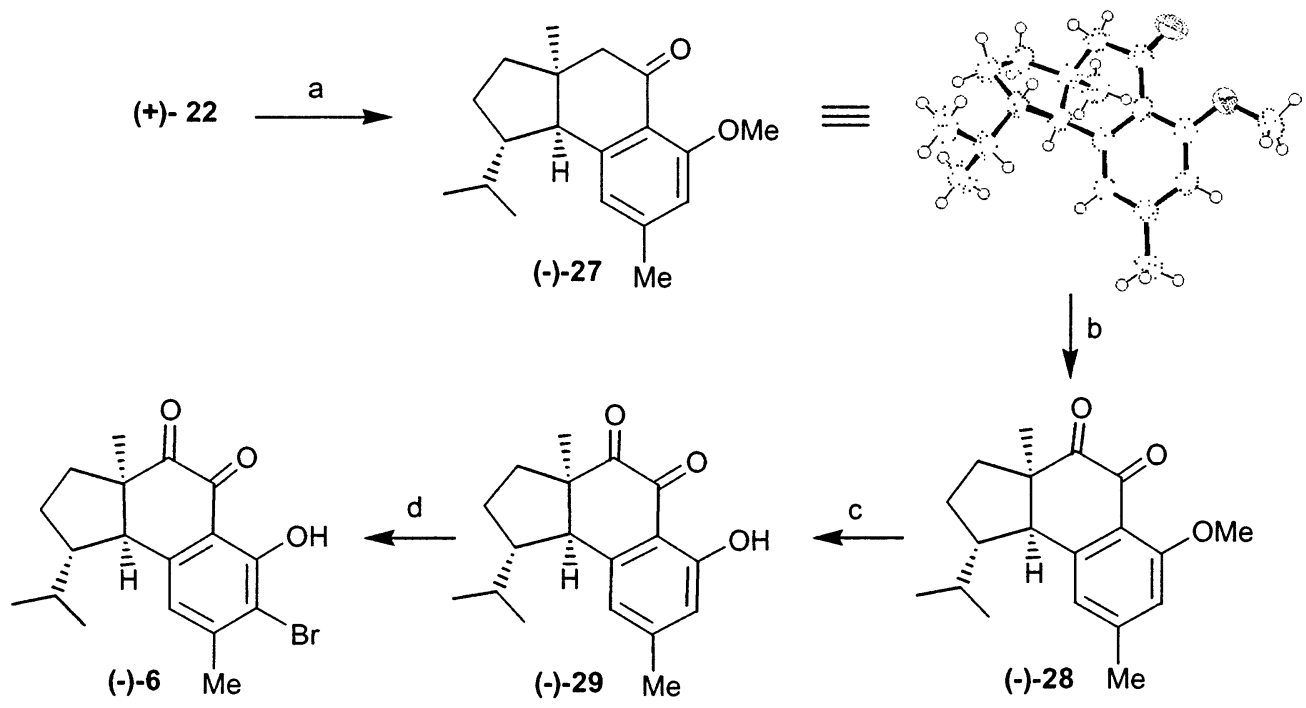

Scheme 4. Reagents and conditions: (a) $10 \% \mathrm{Pd} / \mathrm{C}, \mathrm{H}_{2}, 5$ h, quant.; (b) $\mathrm{SeO}_{2}, \mathrm{AcOH}$ (cat.), aq. dioxane, reflux, $24 \mathrm{~h}, 80 \%$; (c) $\mathrm{BBr}$, DCM, $-20^{\circ} \mathrm{C}, 5 \mathrm{~h}, 90 \%$; (d) NBS, DIPA (cat.), DCM, $0^{\circ} \mathrm{C}, 3 \mathrm{~h}, 90 \%$ 
In summary, we have outlined an enantiospecific approach for the construction of the tricyclic benzofused hydrindane framework present in the hamigeran marine natural products. Starting from $R-(+)$-limonene as the chiron and employing the Heck coupling between aryl triflates and an alkene embedded in a sterically compressed environment, a synthesis of 6-epi(-)-hamigeran B has been accomplished. The general strategy outlined here is being tactically adapted for the synthesis of hamigeran natural products.

\section{Acknowledgements}

One of us (H.M.S.) thanks CSIR for the award of a research fellowship.

\section{References}

1. Faulkner, D. J. Nat. Prod. Rep. 2002, 19, 1 and earlier contributions by the same author in this review series.

2. Wellington, K. D.; Cambie, R. C.; Rutledge, P. S.; Bergquist, P. R. J. Nat. Prod. 2000, 63, 79.

3. Cambie, R. C.; Rickard, C. E. F.; Rutledge, P. S.; Wellington, K. D. Acta Crystallogr. Sect. C: Crystal Structure Commun. 2001, C57, 958.

4. (a) Nicolaou, K. C.; Gray, D.; Tae, J. Angew Chem., Int. Ed. 2002, 40, 3675; (b) Nicolaou, K. C.; Gray, D.; Tae, J. Angew Chem., Int. Ed. 2002, 40, 3679.

5. (a) Mehta, G.; Krishna Murthy, N.; Karra, S. R. J. Am. Chem. Soc. 1991, 113, 5765; (b) Mehta, G.; Karra, S. R. J. Chem. Soc., Chem. Commun. 1991, 1367; (c) Mehta, G.; Karra, S. R.; Krishna Murthy, N. Tetrahedron Lett. 1994, 35, 2761

6. Hwu, J. R.; Leu, L.-C.; Robl, J. A.; Anderson, D. A.; Wetzel, J. M. J. Org. Chem. 1987, 52, 188.

7. All new compounds reported here were characterized on the basis of spectroscopic data (IR, ${ }^{1} \mathrm{H}$ and ${ }^{13} \mathrm{C}$ NMR and mass). Spectral data for some of the key compounds follows. 9: $[\alpha]_{\mathrm{D}}^{24}=-98.7$ ( c 2.3, $\left.\mathrm{CHCl}_{3}\right)$; IR $\left(\mathrm{cm}^{-1}\right) 2959$, 2874, 1732; ${ }^{1} \mathrm{H}$ NMR $\left(300 \mathrm{MHz}, \mathrm{CDCl}_{3}\right): \delta 4.93(\mathrm{t}$, $J=5.1 \mathrm{~Hz}, 1 \mathrm{H}), 3.98-3.74(\mathrm{~m}, 4 \mathrm{H}), 2.20-2.14(\mathrm{~m}, 2 \mathrm{H})$, 2.03-2.00 (m, 2H), $1.86(\mathrm{~d}, J=5.1 \mathrm{~Hz}, 2 \mathrm{H}), 1.79-1.64(\mathrm{~m}$, $2 \mathrm{H}), 0.99(\mathrm{~d}, J=6.6 \mathrm{~Hz}, 3 \mathrm{H}), 0.96(\mathrm{~s}, 3 \mathrm{H}), 0.81(\mathrm{~d}, J=6.3$ $\mathrm{Hz}, 3 \mathrm{H}) ;{ }^{13} \mathrm{C}$ NMR $\left(75 \mathrm{MHz}, \mathrm{CDCl}_{3}\right): \delta 222.45,102.50$, $64.71,64.35,55.20,46.73,40.84,32.98,26.99,21.98$, $21.09,20.43,18.24 ; \mathrm{MS}(\mathrm{ES}): m / z[\mathrm{M}+\mathrm{Na}]^{+}=249 ; 11$ : $[\alpha]_{\mathrm{D}}^{23}=-18.0\left(c 2.5, \mathrm{CHCl}_{3}\right) ; \mathrm{IR}\left(\mathrm{cm}^{-1}\right) 2958,2868,2726$, 1724, 1644; ${ }^{1} \mathrm{H}$ NMR $\left(300 \mathrm{MHz}, \mathrm{CDCl}_{3}\right): \delta 9.73(\mathrm{t}$, $J=1.5 \mathrm{~Hz}, 1 \mathrm{H}), 5.22(\mathrm{~s}, 1 \mathrm{H}), 2.37-2.20(\mathrm{~m}, 5 \mathrm{H}), 1.90$ $1.82(\mathrm{~m}, 1 \mathrm{H}), 1.77-1.67(\mathrm{~m}, 1 \mathrm{H}), 1.12(\mathrm{~s}, 3 \mathrm{H}), 1.00(\mathrm{~d}$, $J=6.6 \mathrm{~Hz}, 6 \mathrm{H}) ;{ }^{13} \mathrm{C}$ NMR $\left(75 \mathrm{MHz}, \mathrm{CDCl}_{3}\right): \delta 203.84$, $150.66,128.90,54.84,46.58,37.32,32.13,29.61,27.47$, 21.37, 21.33; MS (70 eV, EI): $m / z[(\mathrm{M}-28)+1]^{+}=139 ; \mathbf{1 5}$ : $[\alpha]_{\mathrm{D}}^{24}=+23.0\left(c 1.0, \mathrm{CHCl}_{3}\right) ; \mathrm{IR}\left(\mathrm{cm}^{-1}\right) 2957,1710,1693$, 1644; ${ }^{1} \mathrm{H}$ NMR $\left(300 \mathrm{MHz}, \mathrm{CDCl}_{3}\right): \delta 7.21(\mathrm{t}, J=8.4 \mathrm{~Hz}$, $1 \mathrm{H}), 6.73(\mathrm{~d}, J=8.4 \mathrm{~Hz}, 1 \mathrm{H}), 6.57(\mathrm{~d}, J=8.4 \mathrm{~Hz}, 1 \mathrm{H})$, $5.27(\mathrm{~s}, 1 \mathrm{H}), 5.13(\mathrm{~s}, 2 \mathrm{H}), 3.78(\mathrm{~s}, 3 \mathrm{H}), 3.45(\mathrm{~s}, 3 \mathrm{H}), 2.85$ (ABq, $J=16.2 \mathrm{~Hz}, 2 \mathrm{H}), 2.26-2.19(\mathrm{~m}, 3 \mathrm{H}), 1.92-1.83(\mathrm{~m}$, $1 \mathrm{H}), 1.78-1.69(\mathrm{~m}, 1 \mathrm{H}), 1.19(\mathrm{~s}, 3 \mathrm{H}), 0.98(\mathrm{~d}, J=7.2 \mathrm{~Hz}$, $3 \mathrm{H}), 0.97(\mathrm{~d}, J=6.6 \mathrm{~Hz}, 3 \mathrm{H}) ;{ }^{13} \mathrm{C}$ NMR $(75 \mathrm{MHz}$,
$\left.\mathrm{CDCl}_{3}\right): \delta 204.19,156.60,154.05,148.77,130.51,130.23$, $122.50,107.33,104.83,94.57,56.21,55.71,55.60,47.06$, $38.05,31.75,29.56,25.81,21.33(2 \mathrm{C}) ; \mathbf{1 6}:[\alpha]_{\mathrm{D}}^{23}=+21.8(c$ 2.2, $\left.\mathrm{CHCl}_{3}\right)$; IR $\left(\mathrm{cm}^{-1}\right) 2958,1708,1608 ;{ }^{1} \mathrm{H}$ NMR $(300$ $\left.\mathrm{MHz}, \mathrm{CDCl}_{3}\right): \delta 6.55(\mathrm{~s}, 1 \mathrm{H}), 6.38(\mathrm{~s}, 1 \mathrm{H}), 5.25(\mathrm{~s}, 1 \mathrm{H})$, $5.12(\mathrm{~s}, 2 \mathrm{H}), 3.76(\mathrm{~s}, 3 \mathrm{H}), 3.45(\mathrm{~s}, 3 \mathrm{H}), 2.84(\mathrm{~s}, 2 \mathrm{H})$, $2.32-2.25(\mathrm{~m}, 3 \mathrm{H}), 2.25-2.23(\mathrm{~m}, 3 \mathrm{H}), 1.91-1.82(\mathrm{~m}, 1 \mathrm{H})$, $1.75-1.68(\mathrm{~m}, 1 \mathrm{H}), 1.17(\mathrm{~s}, 3 \mathrm{H}), 0.97(\mathrm{~d}, J=6.9 \mathrm{~Hz}, 3 \mathrm{H})$, $0.96(\mathrm{~d}, J=7.2 \mathrm{~Hz}, 3 \mathrm{H}) ;{ }^{13} \mathrm{C}$ NMR $\left(75 \mathrm{MHz}, \mathrm{CDCl}_{3}\right): \delta$ $204.39,156.55,154.07,148.70,140.92,130.61,119.90$, $108.01,105.75,94.54,56.26,55.68$ (2C), 47.18, 38.03, $31.76,29.59,25.86,22.11,21.37$ (2C); HRMS calcd for $\mathrm{C}_{21} \mathrm{H}_{30} \mathrm{O}_{4}[\mathrm{M}+\mathrm{Na}]^{+}: 369.2042$ found: $369.2055 ; \mathbf{1 7}:[\alpha]_{\mathrm{D}}^{24}=$

+24.0 (c 1.0, $\left.\mathrm{CHCl}_{3}\right)$; IR $\left(\mathrm{cm}^{-1}\right) ;{ }^{1} \mathrm{H}$ NMR $(300 \mathrm{MHz}$, $\left.\mathrm{CDCl}_{3}\right): \delta 7.84-7.75(\mathrm{~m}, 2 \mathrm{H}), 7.71(\mathrm{~d}, J=8.4 \mathrm{~Hz}, 1 \mathrm{H})$, 7.49-7.35 (m, 3H), $5.32(\mathrm{~s}, 1 \mathrm{H}), 5.30(\mathrm{~s}, 2 \mathrm{H}), 3.52(\mathrm{~s}, 3 \mathrm{H})$, $3.07(\mathrm{~s}, 2 \mathrm{H}), 2.36-2.18(\mathrm{~m}, 3 \mathrm{H}), 1.99-1.90(\mathrm{~m}, 1 \mathrm{H})$, $1.80-1.70(\mathrm{~m}, 1 \mathrm{H}), 1.27(\mathrm{~s}, 3 \mathrm{H}), 0.97(\mathrm{~d}, J=6.9 \mathrm{~Hz}, 3 \mathrm{H})$, $0.94(\mathrm{~d}, J=7.2 \mathrm{~Hz}, 3 \mathrm{H}) ;{ }^{13} \mathrm{C} \mathrm{NMR}\left(75 \mathrm{MHz}, \mathrm{CDCl}_{3}\right): \delta$ $206.95,151.09,149.20,130.87,130.35,130.26,129.54$ (2C), 128.09, 127.29, 124.38, 123.88, 115.74, 95.11, 56.42, $55.89,47.36,38.03,31.80,29.59,26.50,21.33,21.30 ; \mathbf{2 1}$ : $[\alpha]_{\mathrm{D}}^{24}=+26.3\left(c 0.8, \mathrm{CHCl}_{3}\right) ; \mathrm{IR}\left(\mathrm{cm}^{-1}\right) 2958,1678 ;{ }^{1} \mathrm{H}$ NMR $\left(300 \mathrm{MHz}, \mathrm{CDCl}_{3}\right): \delta 7.43(\mathrm{t}, J=8.1 \mathrm{~Hz}, 1 \mathrm{H}), 6.90$ $(\mathrm{d}, J=7.8 \mathrm{~Hz}, 1 \mathrm{H}), 6.85(\mathrm{~d}, J=8.4 \mathrm{~Hz}, 1 \mathrm{H}), 5.40(\mathrm{~s}, 1 \mathrm{H})$, $3.90(\mathrm{~s}, 3 \mathrm{H}), 3.63(\mathrm{~s}, 1 \mathrm{H}), 2.82(\mathrm{~d} 1 / 2 \mathrm{ABq}, J=14.4 \mathrm{~Hz}$, $1 \mathrm{H}), 2.37(\mathrm{~d} 1 / 2 \mathrm{ABq}, J=14.4 \mathrm{~Hz}, 1 \mathrm{H}), 2.23-2.15(\mathrm{~m}, 2 \mathrm{H})$, $1.63(\mathrm{~s}, 1 \mathrm{H}), 1.25(\mathrm{~s}, 3 \mathrm{H}), 1.11(\mathrm{~d}, J=6.6 \mathrm{~Hz}, 3 \mathrm{H}), 0.79$ $(\mathrm{d}, J=6.6 \mathrm{~Hz}, 3 \mathrm{H}) ;{ }^{13} \mathrm{C}$ NMR $\left(75 \mathrm{MHz}, \mathrm{CDCl}_{3}\right): \delta$ $198.33,159.22,151.48,145.30 \quad(2 \mathrm{C}), 133.49,122.20$, $121.09,109.82,57.06,55.94,50.91,45.28,44.20,27.16$, 26.12, 22.27, 21.68; 22: $[\alpha]_{\mathrm{D}}^{25}=+145.6\left(c 0.9, \mathrm{CHCl}_{3}\right) ; \mathrm{IR}$ $\left(\mathrm{cm}^{-1}\right)$ 2956, 2923, 1681; ${ }^{1} \mathrm{H}$ NMR $\left(300 \mathrm{MHz}, \mathrm{CDCl}_{3}\right): \delta$ $6.70(\mathrm{~s}, 1 \mathrm{H}), 6.66(\mathrm{~s}, 1 \mathrm{H}), 5.40(\mathrm{~s}, 1 \mathrm{H}), 3.89(\mathrm{~s}, 3 \mathrm{H}), 3.57$ (s, $1 \mathrm{H}), 2.81\left(\mathrm{~d} 1 / 2 \mathrm{AB}_{\mathrm{q}}, J=14.1 \mathrm{~Hz}, 1 \mathrm{H}\right), 2.38(\mathrm{~s}, 3 \mathrm{H})$, $2.34-2.17(\mathrm{~m}, 4 \mathrm{H}), 1.23$ (s, 3H), $1.12(\mathrm{~d}, J=7.2 \mathrm{~Hz}, 3 \mathrm{H})$, $0.78(\mathrm{~d}, J=6.6 \mathrm{~Hz}, 3 \mathrm{H}) ;{ }^{13} \mathrm{C}$ NMR $\left(75 \mathrm{MHz}, \mathrm{CDCl}_{3}\right): \delta$ $198.42,159.48,151.33,145.23,144.51,122.98,121.02$, $119.54,110.67,57.05,55.85,50.58,45.19,44.41,27.19$, 25.71, 22.29, 22.19, 21.76; HRMS calcd for $\mathrm{C}_{19} \mathrm{H}_{24} \mathrm{O}_{2}$ $[\mathrm{M}+\mathrm{Na}]^{+}: 307.1674$ found: $307.1683 ; \mathbf{2 7}: \mathrm{mp} 96.0-96.8^{\circ} \mathrm{C}$; $[\alpha]_{\mathrm{D}}^{24}=-16.0\left(c 0.5, \mathrm{CHCl}_{3}\right)$; IR $\left(\mathrm{cm}^{-1}\right) 2952,1676 ;{ }^{1} \mathrm{H}$ NMR $\left(300 \mathrm{MHz}, \mathrm{CDCl}_{3}\right): \delta 6.64(\mathrm{~s}, 1 \mathrm{H}), 6.53(\mathrm{~s}, 1 \mathrm{H})$, $3.89(\mathrm{~s}, 3 \mathrm{H}), 2.71\left(\mathrm{~d} 1 / 2 \mathrm{AB}_{\mathrm{q}}, J=16.5 \mathrm{~Hz}, 1 \mathrm{H}\right), 2.47(\mathrm{~d}$, $J=10.8 \mathrm{~Hz}, 1 \mathrm{H}), 2.36(\mathrm{~s}, 3 \mathrm{H}), 2.27\left(\mathrm{~d} 1 / 2 \mathrm{AB}_{\mathrm{q}}, J=16.5\right.$ $\mathrm{Hz}, 1 \mathrm{H}), 2.05-1.88(\mathrm{~m}, 2 \mathrm{H}), 1.76-1.44(\mathrm{~m}, 4 \mathrm{H}), 1.06(\mathrm{~s}$, $3 \mathrm{H}), 0.97(\mathrm{~d}, J=6.6 \mathrm{~Hz}, 3 \mathrm{H}), 0.86(\mathrm{~d}, J=6.6 \mathrm{~Hz}, 3 \mathrm{H}) ;{ }^{13} \mathrm{C}$ NMR $\left(75 \mathrm{MHz}, \mathrm{CDCl}_{3}\right): \delta 197.91,160.20,148.15$, 144.86, 122.75 (2C), 110.72, 55.86, 52.90, 52.75, 48.71, $44.51,39.48,27.11,25.81,22.53,22.24,22.17,15.28$; HRMS calcd for $\mathrm{C}_{19} \mathrm{H}_{26} \mathrm{O}_{2}[\mathrm{M}+\mathrm{Na}]^{+}: 309.1830$ found: $309.1845 ; 28: \mathrm{mp} 154.5-155.3^{\circ} \mathrm{C} ;[\alpha]_{\mathrm{D}}^{25}=-381.3$ (c 0.8 , $\left.\mathrm{CHCl}_{3}\right)$; IR $\left(\mathrm{cm}^{-1}\right) 2955,1724,1676 ;{ }^{1} \mathrm{H}$ NMR (300 $\left.\mathrm{MHz}, \mathrm{CDCl}_{3}\right): \delta 6.72(\mathrm{~s}, 1 \mathrm{H}), 6.64(\mathrm{~s}, 1 \mathrm{H}), 3.93(\mathrm{~s}, 3 \mathrm{H})$, $2.70(\mathrm{~d}, J=10.2 \mathrm{~Hz}, 1 \mathrm{H}), 2.61-2.56(\mathrm{~m}, 1 \mathrm{H}), 2.42(\mathrm{~s}, 3 \mathrm{H})$, $1.74-1.47(\mathrm{~m}, 5 \mathrm{H}), 1.22(\mathrm{~s}, 3 \mathrm{H}), 0.90(\mathrm{~d}, J=6.6 \mathrm{~Hz}, 3 \mathrm{H})$, $0.77(\mathrm{~d}, J=6.9 \mathrm{~Hz}, 3 \mathrm{H}) ;{ }^{13} \mathrm{C}$ NMR $\left(75 \mathrm{MHz}, \mathrm{CDCl}_{3}\right): \delta$ $200.36,179.19,161.83,147.44,147.14,122.94,118.63$, $111.18,57.64,56.57,55.99,55.14,33.82,27.17,22.49$, 22.39, 21.93, 21.83, 15.58; HRMS calcd for $\mathrm{C}_{19} \mathrm{H}_{24} \mathrm{O}_{2}$ $[\mathrm{M}+\mathrm{Na}]^{+}: 323.1623$ found: $323.1642 ; 6$ : $\mathrm{mp} 218-220^{\circ} \mathrm{C}$; $[\alpha]_{\mathrm{D}}^{25}=-220.0\left(c 0.2, \mathrm{CHCl}_{3}\right) ; \mathrm{IR}\left(\mathrm{cm}^{-1}\right) 2956,2924,1720$; ${ }^{1} \mathrm{H}$ NMR $\left(300 \mathrm{MHz}, \mathrm{CDCl}_{3}\right): \delta 12.86(\mathrm{~s}, 1 \mathrm{H}), 6.68(\mathrm{~s}$, 
1H), 2.80-2.71 (m, 4H), 2.54 (s, 3H), 2.39 (s, 1H), 1.71-1.46 $(\mathrm{m}, 2 \mathrm{H}), 1.26(\mathrm{~s}, 3 \mathrm{H}), 0.94(\mathrm{~d}, J=6.6 \mathrm{~Hz}, 3 \mathrm{H}), 0.80$ (d, $J=6.9 \mathrm{~Hz}, 3 \mathrm{H}) ;{ }^{13} \mathrm{C} \mathrm{NMR}\left(75 \mathrm{MHz}, \mathrm{CDCl}_{3}\right): \delta 198.30$, $183.64,161.42,150.33,143.90,122.98,115.58,112.04$, 59.15, 56.78, 54.93, 32.60, 26.83, 24.54, 21.89, 21.73, 21.50, 15.10; HRMS calcd for $\mathrm{C}_{18} \mathrm{H}_{21} \mathrm{BrO}_{3}[\mathrm{M}+\mathrm{Na}]^{+}: 387.0572$ found: 387.0583 .

8. Link, J. T. Org. React. 2002, 60, 157.

9. Winkle, M. R.; Ronald, R. C. J. Org. Chem. 1982, 47, 2101.

10. (a) Ritter, K. Synthesis 1993, 735; (b) Cabri, W.; Candiani, I.; DeBernardinis, S.; Francalanci, F.; Penco, S. J. Org. Chem. 1991, 56, 5796.

11. Crystal data for 27: X-ray data were collected at $293 \mathrm{~K}$ on a SMART CCD-BRUKER diffractometer with graphite monochromated $\operatorname{MoK} \alpha$ radiation $(\lambda=0.7107 \AA)$. The structure was solved by direct methods (SIR-92). Refinement was by full-matrix least-squares procedures on $F^{2}$ using SHELXL-97. The non-hydrogen atoms were refined anisotropically whereas hydrogen atoms were refined isotropically. Compound 27: $\mathrm{C}_{19} \mathrm{H}_{26} \mathrm{O}_{2}, \mathrm{MW}=286$, colorless crystal, crystal system: orthorhombic, space group $P 2_{1} 2_{1} 2_{1}$ : cell parameters: $a=8.467$ (2), $b=10.089$ (2), $c=19.267$ (5) $\AA, V=1645.97 \AA^{3}, Z=4, D_{\text {calcd }}=1.156 \mathrm{~g}$ $\mathrm{cm}^{-3}, F(000)=624.0, \mu=0.07 \mathrm{~mm}^{-1}$. Total number of least-squares parameters $=294, R_{1}=0.0506$ for $2530 \mathrm{~F}_{\mathrm{o}}$ $>4 \sigma\left(F_{\mathrm{o}}\right)$ and 0.0736 for all 3367 data. $w R_{2}=0.1080$, $\mathrm{GOF}=1.072$, restrained $\mathrm{GOF}=1.072$ for all data. Crystallographic data (without structure factors) have deposited with the Cambridge Crystallographic Data Center (CCDC 212686). An ORTEP diagram of compound 27 with 50\% ellipsoidal probability is shown in Scheme 4. 